\title{
Correlative Light and Electron Imaging of Cell-Cell Interactions within the Islet of Langerhans
}

\author{
David W. Piston ${ }^{1}$, Jing Hughes ${ }^{1}$, Alessandro Ustione ${ }^{1}$ \\ ${ }^{1}$ Department of Cell Biology and Physiology, Washington University, St Louis MO 63110
}

The islet of Langerhans plays a central role in blood glucose homeostasis through dose-dependent and regulated hormone secretion. The discovery of insulin and its utilization for diabetes therapies ranks among the most important medical advances of the $20^{\text {th }}$ century, leading research to be focused on the insulin-secreting $\beta$-cells. The actions of another islet hormone, glucagon, are counter-productive in the treatment of diabetes, although the importance of the glucagon-secreting $\alpha$-cells is becoming increasingly clear. During hypoglycemia, the $\alpha$-cells secrete glucagon that stimulates glucose output from the liver, whereas under euglycemia, glucagon release is suppressed.

$\alpha$-cell function remains poorly understood [1], but it is generally assumed that $\alpha$-cell regulation requires the juxtaposed presence of either $\beta$-cells, $\delta$-cells, or both. The need for juxtaposed $\beta$-cells is evidenced by the loss of glucose inhibition of glucagon secretion when $\beta$-cell function is eliminated in diabetes. Similarly, islet glucagon secretion is disrupted when the secretory product of islet $\delta$ cells, somatostatin (SST), is deleted. However, neither insulin nor SST alone inhibit glucagon secretion from dispersed $\alpha$-cells [2], consistent with the finding that glucose inhibition of glucagon secretion is lost in dispersed $\alpha$-cells. The mechanism(s) proposed to regulate of glucagon secretion remain controversial, but we propose that at least part of the regulation arises from cell-cell contact mediated by ephrin-A-EphA signaling [3]. EphA4 is the main ephrin receptor expressed in $\alpha$-cells, which suggests that it may play an important role in regulating glucagon secretion. Islet cells are also heavily connected via gap junctions, which are intercellular channels that allow the direct transfer of ions and second messengers between adjacent cells. While the electrical coupling between $\beta$-cells is well-established [4], gap junction coupling of $\alpha$-cells has not been reported despite early evidence pointing to gap junctions between $\alpha$ - and $\beta$-cells [5]. Curiously, genetic deletion of $\beta$-cell gap junction proteins leads to deficient glucagon secretion, which leads us to consider whether the ultrastructural arrangements of the $\alpha$-/ $\beta$-cell interface plays a role in regulating $\alpha$-cell function.

Structural images can be obtained at nanometer resolution with electron microscopy, but the invasive character of the electron irradiation precludes dynamic EM measurements on live cells. As suggested above, though, the nanostructural context and high-resolution localization of biomolecules within this context is crucial for understanding function and malfunction in cellular and biomolecular processes. On the other hand, fluorescence microscopy permits dynamic live imaging, but falls short in terms of molecular resolution. To resolve the specific molecular motions and functional distributions, we are using electron microscopy (EM), and correlative fluorescence with EM. Approaches that we are currently using include correlative fluorescence and SEM on intact cells in water as we have recently published [6] and FIB-SEM three-dimensional EM that we are utilizing in a correlative manner with 3D fluorescence imaging. An example of this work is 
shown in Fig. 1. However, an exciting possibility that is ideal for this application is the use of STEM z-contrast of small gold labels with cryotomography of intact cells or tissue sections. We have used this approach in live cells and with fixed cells in aqueous media [7], and an example of this work is shown in Fig. 2, which show nm level resolution through $>1 \mu \mathrm{m}$ of aqueous buffer. We have calculated that small gold nanoparticles (nanogold, which is $67 \mathrm{Au}$, or even smaller $31 \mathrm{Au}$ atom clusters) can be measured by z-contrast through several $\mu \mathrm{m}$ of aqueous buffer.

\section{References:}

[1] Gylfe, E., Ups J Med Sci, 2016. 121(2): p. 120-32.

[2] Le Marchand, S.J. and D.W. Piston, J Biol Chem, 2010. 285(19): p. 14389-98.

[3] Benninger, R.K., et al., J Physiol, 2011. 589(Pt 22): p. 5453-66.

[4] Benninger, R.K. and D.W. Piston, Trends Endocrinol Metab, 2014. 25(8): p. 399-406.

[5] Orci, L., et al., J Clin Invest, 1975. 56(4): p. 1066-70.

[6] Liv, N., et al., ACS Nano, 2016. 10(1): p. 265-73.
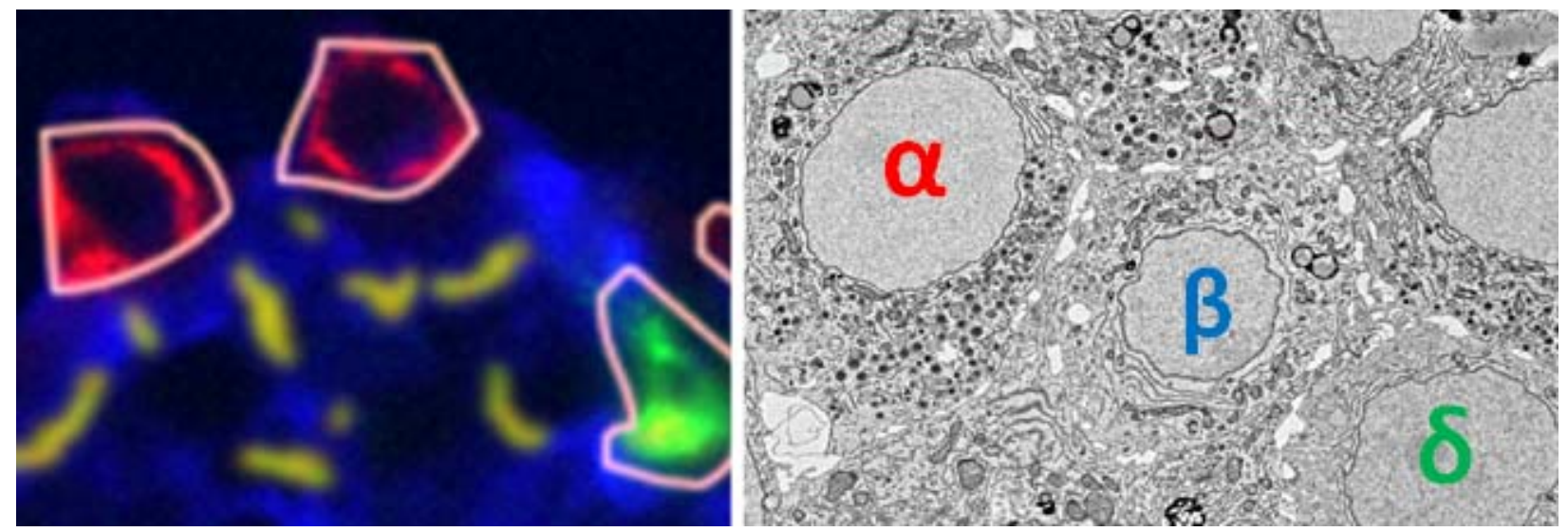

Figure 1. Correlative Fluorescence/Electron Microscopy. (Left) Immunofluorescence of part of an islet with $\alpha$-cells (red), $\beta$-cells (blue), $\delta$-cells (green), and gap junctions (yellow). (Right) Single FIB-SEM image of the interface between the three cell types.

[7] de Jonge, N., et al., Proc Nat Acad Sci USA, 2009. 106(7): p. 2159-64
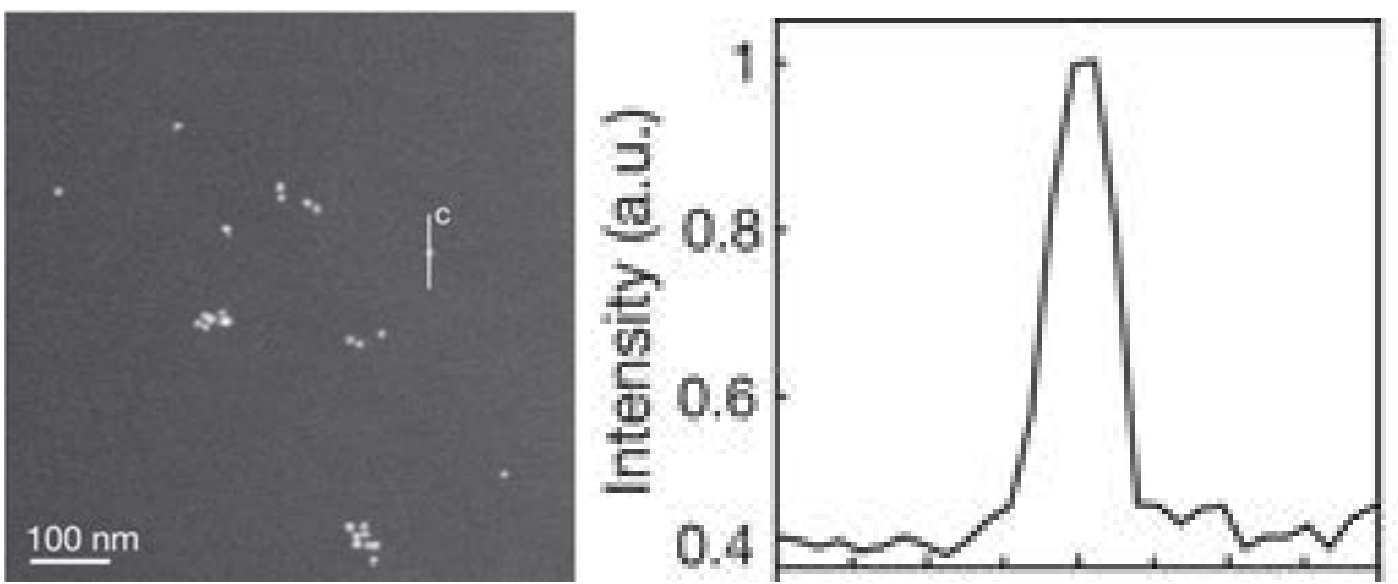

Figure 2. Resolution in Liquid STEM. (Left) Image of a COS7 cell edge labeled with EGF-Au. (Right) Line-scan over a single gold nanoparticle indicated by the line " $c$ ". 